Research Article

\title{
Carbon Emission Reduction Decision and Revenue-Sharing Contract with Consumers' Low-Carbon Preference and CER Cost under Carbon Tax
}

\author{
Chao-qun Han $\mathbb{D}^{1},{ }^{1,2}$ Hua-ying Gu $\mathbb{D}^{2},{ }^{2}$ Li-hui Sui, ${ }^{1}$ and Chang-peng Shao ${ }^{3}$ \\ ${ }^{1}$ School of Business, Shanghai Dianji University, Shanghai 201306, China \\ ${ }^{2}$ Logistics Research Center, Shanghai Maritime University, Shanghai 201306, China \\ ${ }^{3}$ School of, Shanghai Dianji University, Shanghai 201306, China \\ Correspondence should be addressed to Hua-ying Gu; ghy_hust@163.com
}

Received 17 March 2020; Revised 14 December 2020; Accepted 13 April 2021; Published 8 May 2021

Academic Editor: Biswajit Sarkar

Copyright ( 2021 Chao-qun Han et al. This is an open access article distributed under the Creative Commons Attribution License, which permits unrestricted use, distribution, and reproduction in any medium, provided the original work is properly cited.

Since the tax of carbon emission is popular and consumers are exhibiting low-carbon preference, the green manufactures have to spend more extra cost on investing carbon emission reduction (CER) technology to decrease the carbon emission. To encourage the manufacture's CER investment efforts, this paper explores the impact of carbon tax, CER cost, and consumers' low-carbon preference on low-carbon decision-making and designs a revenue-sharing contract (RS) by constructing Stackelberg models. Based on the theoretical and numerical analysis, this paper finds that the supply chain would benefit from the increment of consumer's environmental awareness but be depressed by the increase of the CER investment cost factor. Additionally, there exists a unique optimal carbon tax to make CER degree the maximum. Furthermore, RS can effectively promote manufacturers to reduce carbon emissions and also improve the supply chain efficiency.

\section{Introduction}

With the rapid development of the economy, global carbon dioxide emission has sharply increased in the past few decades, which significantly resulted in serious climate and global warming [1]. It is an urgent task to reduce carbon emissions to allay global warming. And this task has drawn high attention from all social members. Governments around the world have promulgated and implemented relevant carbon policies to reduce carbon emissions in practice, such as carbon tax, cap and trade, and low-carbon offset. Among these policies, carbon tax is considered an effective environmental policy in China. In addition, more and more consumers are exhibiting low-carbon preference, that is, consumers are willing to pay higher prices for the same function but more green products [2, 3]. To comply with governments' regulatory schemes and meet the need of low-carbon preference customers, many manufacturers begin to employ the CER investment and adjust their existing production mode to produce low-carbon products. However, the manufacturer must take extra cost to employ the CER investment. The high cost of the CER investment brings the challenge to the manufacturer. Whether the increased revenue with the CER investment can balance or compensate for the additional cost is the focus of attention for the manufacturers. Therefore, it is important that government's carbon tax, customers' low-carbon preference, and CER investment cost should be considered by the manufacturer in making strategic decisions. Moreover, it is necessary to create an incentive mechanism to urge manufactures to explore CER technology investment.

The extant findings have shown that coordination contracts or carbon tax as the mechanism are used to compensate and counterbalance the CER cost, resulting in motiving the manufacture to employ CER investments $[4,5]$. However, little consideration has been given to the effects of low-carbon preference, CER cost factor, and carbon tax simultaneously on the design of coordination 
contracts. In this context, from the perspective of encouraging manufacturers to reduce carbon emissions, this study tries to address the following questions: (1) how do the manufacturer and the retailer make decisions considering the carbon tax, customers' low-carbon preference, and CER cost factor simultaneously? (2) How to set appropriate carbon tax that encourages the manufacturer reduce carbon emissions? (3) How to design a suitable coordinate contract to improve CER degree and enhance supply chain efficiency?

To answer the above questions, this paper considers a low-carbon supply chain, where the manufacturer makes CER investment to produce low-carbon products and sell them through a single retailer. In modeling and analysis, this paper finds the equilibrium strategies of the supply chain under centralized and decentralized decision-making and discusses the impacts of low-carbon awareness, CER cost factor, and carbon tax on the supply chain in detail. Furthermore, a revenue-sharing contract (RS) is adopted to stimulate the manufacturer to improve CER degree and coordinate the supply chain. The results indicate that the supply chain is better off exhibiting low-carbon preference and will suffer a loss from the increase CER cost factor when the carbon tax is low. Moreover, the paper finds that there exists a unique optimal carbon tax to make CER degree the maximum. And RS can encourage the manufacturer's CER investments but fails to coordinate the supply chain, and the manufacturer and the retailer prefer to implement RS when the RS coefficient is in a certain threshold. This research makes three contributions to the literature. First, this paper integrates consumers' low-carbon awareness, CER cost factor, and carbon tax into supply chain models and explores how these factors affect the CER degree and firms' profit. Second, this paper finds that the government should impose different optimal carbon taxes to inspire the manufacturer to make the best efforts on CER investments in different scenarios. Finally, this paper designs RS to incentive the manufacture to improve CER degree and also enhance the supply chain efficiency.

The remainder of this paper is organized as follows. Section 2 briefly reviews the closely related literature studies. Section 3 describes model notations, assumptions, and formulations. The game models are established to obtain the equilibrium solutions in Section 4. A numerical example is conducted to compare the optimal strategies in Section 5. Section 6 provides concluding remarks. In addition, all proofs are provided in Appendix.

\section{Literature Review}

With the transition to the sustainable supply chain, there is a growing interest in the decision-making problems and the design of coordination/incentive contracts. Research on low-carbon supply chain decisions mainly focuses on price [6-8], low-carbon products' quantity $[9,10]$, manufacturing/remanufacturing [11], etc. Low-carbon supply chain coordination has been studied by different mechanisms including RS [12, 13], BB (buyback contract) [14], and CS (cost-sharing contract) $[15,16]$. A brief review of the previous studies that are related to this paper is conducted with regard to low-carbon decision-making of the supply chain and RS design with CER cost and consumers' low-carbon preference and carbon tax. Table 1 compares the research scope of this paper with that of typical studies on CER.

2.1. Low-Carbon Decision-Making of the Supply Chain. Environmental issues are new hotspots in supply chain research. Currently, low-carbon decision-making of the supply chain is mainly studied from three factors: lowcarbon preference, CER cost factor, and carbon tax.

In the view of carbon tax, Mishra et al. [17] developed a carbon cap and tax-regulated sustainable inventory management model. Chen and $\mathrm{Hu}$ [18] used the evolutionary game theory to examine the behavioral strategies of the manufacturers in response to carbon tax. Despite attracting much attention from the academia, the specific quantity or the decision of the optimal carbon tax is still not discussed totally. This paper discusses the optimal carbon tax in different scenarios to encourage the manufacturer to reduce carbon emission. In the view of CER cost, Li et al. [12] examined the impact of the CER cost factor and carbon tax on price and CER decisions. In the view of consumers' lowcarbon preference, Wang et al. [19] developed game models for studying CER considering low-carbon preference in the retailer-dominant and power-balanced cases. Wu et al. [20] proposed a low-carbon decision-making model of online shopping supply chain by considering the low-carbon awareness of online shoppers. Liang and Futou [21] investigated the impact of the low-carbon preference on the optimal joint carbon reduction strategy. Wang et al. [22] found that the CER and the channel profit increase with the increase in the consumers' low-carbon preference in the dual-channel supply chain. Assuming that consumers maintain a low-carbon preference and considering CER cost and carbon tax, this paper investigates the impact of these three factors on the optimal CER and price strategy and designs RS to encourage the manufacturer to reduce carbon emission.

2.2. Revenue-Sharing Contract. Li et al. [7] investigated the impact of the RS contract offered by a retailer on CER. Yu et al. [23] deduced equilibrium emission reduction and pricing strategies in RS considering reference emission and cost learning effects. Yang and Chen [5] studied the impact of RS offered by a retailer on manufacturer's CER effort considering consumers' low-carbon preference and carbon tax simultaneously. Liu et al. [24] examined the effects of RS on product greenness and pricing strategies under different power structures. The above studies have proven that the improvement of greenness of the supply chain is strongly linked to the RS contract among supply chain members.

2.3. Implication Referred. Based on the extant literature studies, many authors analyzed the impact of various factors on the low-carbon operation decision-making of enterprises. However, the aforementioned research studies have incomprehensively considered the impact of CER cost 
TABle 1: Authors' contribution.

\begin{tabular}{|c|c|c|c|c|}
\hline Authors & CER cost & Carbon tax & Consumers' low-carbon preference & Coordination/incentive contract \\
\hline Mishra et al. [17] & & $\checkmark$ & & \\
\hline Chen and $\mathrm{Hu}[18]$ & & $\checkmark$ & & \\
\hline Wang et al. [19] & & & $\checkmark$ & \\
\hline Yang and Chen [5] & & $\checkmark$ & $\checkmark$ & $\checkmark$ \\
\hline Li et al. [12] & $\checkmark$ & & $\checkmark$ & $\checkmark$ \\
\hline This paper & $\checkmark$ & $\sqrt{ }$ & $\checkmark$ & $\checkmark$ \\
\hline
\end{tabular}

factor, carbon tax, and consumers' low-carbon awareness on the low-carbon decisions and RS. Our paper aims to fill the gap by introducing CER cost factor, consumers' low-carbon preference, and carbon tax into the analytical framework. Thus, the incentive effect of carbon tax and RS can be studied more accurately and realistically. The conclusion will help the firms make decisions and design incentive contract in a low-carbon supply chain, as well as provide insights for the government to formulate the optimal carbon tax policy.

\section{Model Description}

3.1. Notations. This paper focuses on a low-carbon supply chain comprising one manufacturer and one retailer where consumers are exhibiting low-carbon preference. The manufacturer produces low-carbon products and sells them to the retailer in the wholesale price. The manufacturer is dominant in the supply chain, who decides the CER degree and the wholesale price. The retailer decides the retail price and sells to consumers. The government imposes carbon tax on carbon emission. Due to the need of the research problem, the following variables and parameters are defined in Table 2.

3.2. Assumptions. For the purposes of discussion and without loss of generality, this paper makes the following basic assumptions.

Assumption 1. It is assumed the demand function is linearly decreasing in selling price and increasing in CER degree [25], that is, the market demand function is described as follows: $D=a-p+\beta\left(e_{1}-e_{0}\right)=a-p+\beta e$.

Assumption 2. The manufacturer takes extra cost to invest CER technologies. It is common knowledge that the manufacturer makes initial changes in products and processes easily while the subsequent improvement being more difficult [7]. Thus, CER cost function is described as follows: $c_{e}=\left(\eta e^{2} / 2\right)$, which means CER cost is a quadratic function of CER degree $e$, which is commonly employed in related works [26].

Assumption 3. The manufacturer must pay the carbon tax for carbon emission in the production process. Every unit carbon emission should pay $t$ carbon tax. Therefore, the manufacturer will pay carbon tax $t e_{1}$ for each low-carbon product.

Assumption 4. In order to ensure all decision variables and profits are positive, we assume $a-c-t e_{0}>0, t<\beta$, and $(\beta+t)^{2}<2 \eta$ throughout the paper.

3.3. Basic Model. Using the aforementioned notations and assumptions, this paper models the profits of the manufacturer, the retailer, and the total supply chain as follows. The manufacturer's profit $\left(\pi_{m}\right)$ includes the sales revenue, production cost, CER investment cost, and carbon tax. The retailer's profit $\left(\pi_{r}\right)$ consists of sales revenue and wholesale cost. The total profit $\left(\pi_{s c}\right)$ for the whole supply chain is the sum of both firms' profit. Note that $D=a-p+\beta\left(e_{0}-e_{1}\right)=a-p+\beta e$. So, we can get the profits $\left(\pi_{m}, \pi_{r}\right)$ of the manufacturer and the retailer. Hence, we formulate $\pi_{m}, \pi_{r}$, and $\pi_{s c}$ individually:

$$
\begin{aligned}
& \pi_{m}=\left[w-c-t e_{1}\right] D-c_{e}=\left[w-c-t e_{1}\right](a-p+\beta e)-\frac{1}{2} \eta e^{2} \\
& \pi_{r}=(p-w)(a-p+\beta e) \\
& \pi_{s c}=\pi_{m}+\pi_{r} .
\end{aligned}
$$

\section{The Models and Results}

4.1. Centralized Supply Chain. As a benchmark, the manufacturer and the retailer jointly determine the CER degree $e^{c}$ and the retail price $p^{c}$ in a centralized supply chain. This paper obtains the profit function of the centralized supply chain $\pi_{s c}^{c}$ as follows. The superscript $c$ is used to denote the centralized scenario.

$$
\pi_{s c}^{c}=\left[p-c-t\left(e_{0}-e\right)\right](a-p+\beta e)-\frac{\eta e^{2}}{2} .
$$

Proposition 1. In a centralized supply chain, the optimal CER degree is $\left(e^{c *}\right)$, the retail price is $\left(p^{c *}\right)$, and the maximum supply chain profit is $\left(\pi_{s c}^{c *}\right)$ : 
TABLE 2: Notation and parameters.

\begin{tabular}{lc}
\hline Notation & Interpretation \\
\hline $\begin{array}{l}\text { Decision variables } \\
p\end{array}$ & Wholesale price \\
Retail price & Carbon emission reduction degree \\
& $e=e_{1}-e_{0}$
\end{tabular}

$$
\begin{aligned}
e^{c *} & =\frac{\left(a-c-t e_{0}\right)(\beta+t)}{\left[2 \eta-(\beta+t)^{2}\right]}, \\
p^{c *} & =\frac{\left[\left(a+c+t e_{0}\right)(\eta-\beta t)-\beta^{2} c-\beta^{2} t e_{0}-t^{2} \alpha\right]}{\left[2 \eta-(\beta+t)^{2}\right]}, \\
\pi_{s c}^{c *} & =\frac{-\eta\left(c-\alpha+e_{0} t\right)^{2}}{2\left(\beta^{2}+2 \beta t+t^{2}-2 \eta\right)} .
\end{aligned}
$$

Proof. See Appendix.

Lemma 1. In a centralized supply chain,

(1) $\left(\partial e^{c *} / \partial \eta\right)<0,\left(\partial p^{c *} / \partial \eta\right)<0$, and $\left(\partial \pi_{s c}^{c *} / \partial \eta\right)<0$

(2) $\left(\partial e^{c *} / \partial \beta\right)>0,\left(\partial p^{c *} / \partial \beta\right)>0$, and $\left(\partial \pi_{s c}^{c *} / \partial \beta\right)>0$

(3) If $0<t<t^{c *}$, then $\left(\partial e^{c *} / \partial t\right)>0$; if $t^{c *}<t$, then $\left(e^{c *} / \partial t\right)<0$

Proof. See Appendix.

Lemma 1 demonstrates that the optimal CER degree $e^{c *}$, the optimal retail price $p^{c *}$, and the maximum supply chain profit $\pi_{s c}^{c *}$ are decreasing in the CER cost factor $\eta$ and are increasing in the consumers' low-carbon preference $\beta$.

Lemma 1 also states that there is an optimal carbon tax $t^{c *}$ in the centralized supply chain, which makes the CER degree the maximum.

4.2. Decentralized Supply Chain. In a decentralized supply chain, the relationship between the manufacturer and the retailer is modeled as a Stackelberg game, where the manufacturer is the leader and the retailer is the follower. In the first stage, the manufacturer decides the CER degree $e^{d}$ and the wholesale price $w^{d}$. In the second stage, the retailer sets

the retail price $p^{d}$ based on the manufacturer's decisions. The profit functions of the manufacturer and the retailer are formulated as follows. The backward induction is utilized to solve this model for obtaining the optimum solution. The superscript $d$ is used to denote the decentralized scenario.

$$
\begin{aligned}
& \pi_{m}^{d}=\left[w-c-t\left(e_{0}-e\right)\right](a-p+\beta e)-\frac{1}{2} \eta e^{2}, \\
& \pi_{r}^{d}=(p-w)(a-p+\beta e) .
\end{aligned}
$$

Proposition 2. In the decentralized supply chain, the optimal retail price is $\left(p^{d *}\right)$, the optimal wholesale price is $\left(w^{d *}\right)$, the optimal CER degree is $\left(e^{d *}\right)$, and the maximum profit of the manufacturer, the retailer, and the supply chain is $\left(\pi_{m}^{d *}, \pi_{r}^{d *}, \pi_{s c}^{d *}\right)$

$$
\begin{aligned}
e^{d *} & =\frac{\left(a-c-t e_{0}\right)(\beta+t)}{4 \eta-(\beta+t)^{2}} \\
w^{d *} & =\frac{\beta^{2} c-2 c \eta-2 a \eta+a t^{2}+a \beta t+\beta c t-2 e_{0} \eta t+\beta e_{0} t^{2}+\beta^{2} e_{0} t}{\beta^{2}+2 \beta t+t^{2}-4 \eta} \\
p^{d *} & =\frac{\beta^{2} c-c \eta-3 a \eta+a t^{2}+a \beta t+\beta c t-e_{0} \eta t+\beta e_{0} t^{2}+\beta^{2} e_{0} t}{\beta^{2}+2 \beta+t^{2}-4 \eta} \\
\pi_{m}^{d *} & =\frac{-\eta\left(c-a+e_{0} t\right)^{2}}{2\left(-4 \eta+2 \beta t+\beta^{2}+t^{2}\right)} \\
\pi_{r}^{d *} & =\frac{\eta^{2}\left(c-a+e_{0} t\right)^{2}}{\left(-4 \eta+2 \beta t+\beta^{2}+t^{2}\right)^{2}}, \\
\pi_{s c}^{d *} & =\frac{-\eta\left(c-a+e_{0} t\right)^{2}\left(\beta^{2}+2 \beta t+t^{2}-6 \eta\right)}{2\left(-4 \eta+2 \beta t+\beta^{2}+t^{2}\right)^{2}} .
\end{aligned}
$$


Proof. See Appendix.

Lemma 2. In the decentralized supply chain,

(1) $\left(\partial e^{d *} / \partial \eta\right)<0,\left(\partial p^{d *} / \partial \eta\right)<0$, and $\left(\partial \pi_{s c}^{d *} / \partial \eta\right)<0$

(2) $\left(\partial e^{d *} / \partial \beta\right)>0,\left(\partial p^{d *} / \partial \beta\right)>0$, and $\left(\partial \pi_{s c}^{d *} / \partial \beta\right)>0$

(3) If $0<t<t^{d *}$, then $\left(\partial e^{d *} / \partial t\right)>0$; if $t^{d *}<t$, then $\left(e^{d *} / \partial t\right)<0$

Proof. See Appendix.

Lemma 2 demonstrates that the CER degree $e^{d *}$, the retail price $p^{d *}$, and the supply chain profit $\pi_{s c}^{d *}$ will increase as the CER cost factor $\eta$ decreases. Moreover, the CER degree $e^{d *}$, the retail price $p^{d *}$, and the supply chain profit $\pi_{s c}^{d *}$ will increase as the consumers' low-carbon preference $\beta$ increases.

Lemma 2 also states that there is an optimal carbon tax $t^{d *}$ which makes the CER degree the maximum.

According to the calculation and analysis in the above section, we compare the equilibrium outcomes in the centralized and the decentralized supply chain, which are shown in Theorem 1.

Theorem 1. (i) $e^{d *}<e^{c *}$ and $\pi_{s c}^{d *}<\pi_{s c}^{c *}$

(ii) If $\eta<t \beta+\beta^{2}, p^{c *}>p^{d *}$; if $\eta>t \beta+\beta^{2}, p^{c *}<p^{d *}$; if $\eta=t \beta+\beta^{2}, p^{c *}=p^{d *}$

Proof. See Appendix.

Theorem 1 shows that the optimal CER degree and the maximum channel profit in the centralized supply chain are both higher than those in the decentralized supply chain. This phenomenon is called "double marginal effect." The following sections design the RS contract to eliminate the double marginal effect.

4.3. Revenue-Sharing Contract for the Decentralized Supply Chain. In this section, we propose the RS contract in which the manufacturer offers the retailer a lower wholesale price, and the retailer shares revenue with the manufacturer with a fraction $\varphi(0<\varphi \leq 1)$.

The timeline is as follows. Firstly, the manufacturer makes CER efforts to reduce the emission of the products, which yields the CER degree $e^{r s}$ and the wholesale price $w^{r s}$. Secondly, the retailer decides his selling price $p^{r s}$. The profit functions of the manufacturer and the retailer are as follows. They are solvable by using backward induction. The superscript $r s$ is used to denote the RS scenario.

$$
\begin{aligned}
& \pi_{m}^{r s}=\left[w-c-t\left(e_{0}-e\right)+1-\varphi p\right](a-p+\beta e)-\frac{1}{2} \eta e^{2} \\
& \pi_{r}^{r s}=(\varphi p-w)(a-p+\beta e) .
\end{aligned}
$$

Proposition 3. Under the RS contract, the optimal CER degree is $\left(e^{r s *}\right)$, the optimal wholesale price is $\left(w^{r s *}\right)$, the optimal retail price is $\left(p^{r * *}\right)$, and the maximum profit of the manufacturer and the retailer is $\left(\pi_{m}^{r s *}, \pi_{r}^{r s *}\right)$ :

$$
\begin{aligned}
e^{r s *} & =\frac{(\beta+t)\left(a-c-t e_{0}\right)}{2 \eta(1+\varphi)-(\beta+t)^{2}}, \\
w^{r s *} & =\frac{\varphi\left(\beta^{2} c-2 c \eta+a t^{2}+a \beta t+\beta c t-2 a \eta \varphi-2 e_{0} \eta t+\beta e_{0} t^{2}+\beta^{2} e_{0} t\right)}{\beta^{2}+2 t \beta+t^{2}-2 \eta-2 \eta \varphi}, \\
p^{r s *} & =\frac{\beta^{2} c-c \eta-a \eta+a t^{2}+a \beta t+\beta c t-2 a \eta \varphi-e_{0} \eta t+\beta e_{0} t^{2}+\beta^{2} e_{0} t}{\beta^{2}+2 t \beta+t^{2}-2 \varphi \eta-2 \eta}, \\
\pi_{m}^{r s *} & =\frac{-\eta\left(c-a+e_{0} t\right)^{2}}{2\left(\beta^{2}+2 \beta t+t^{2}-2 \eta-2 \eta \varphi\right)} . \\
\pi_{r}^{r s *} & =\frac{\eta^{2} \varphi\left(c-a+e_{0} t\right)^{2}}{\left(-2 \eta+2 \beta t-2 \eta \varphi+\beta^{2}+t^{2}\right)^{2}},
\end{aligned}
$$

The proof is similar to that of Proposition 2 and is hence omitted here for brevity.

Lemma 3. Under RS,
(1) $\left(\partial e^{r s *} / \partial \eta\right)<0,\left(\partial p^{r s *} / \partial \eta\right)<0$, and $\left(\partial \pi_{s c}^{r s *} / \partial \eta\right)<0$

(2) $\left(\partial e^{r s *} / \partial \beta\right)>0,\left(\partial \pi_{s c}^{r * *} / \partial \beta\right)>0$, and $\left(\partial p^{r s *} / \partial \beta\right)>0$

(3) If $0<t<t^{r s *}$, then $\left(\partial e^{r s *} / \partial t\right)>0$; if $t^{r s *}<t$, then $\left(\partial e^{r s *} / \partial t\right)<0$ 
Lemma 3 demonstrates that the CER degree $e^{r s *}$, the retail price $p^{r s *}$, and the supply chain profit $\pi_{s c}^{r s *}$ will increase as the CER cost factor $\eta$ decreases. Moreover, the CER degree $e^{r s *}$, the retail price $p^{r * *}$, and the supply chain profit $\pi_{s c}^{r s *}$ will increase as the consumers' low-carbon preference $\beta$ increases.

Lemma 3 also states that there is an optimal carbon tax $t^{r s *}$ which makes the CER degree the maximum.

The proof is similar to that of Lemma 2 and is hence omitted here for brevity.

Theorem 2. Under the RS contract, $e_{m}^{r s}>e_{m}^{d}$; if $\left(\left[2 \eta-(t+\beta)^{4}\right]^{2} / 4 \eta^{2}\right)<\varphi<1$, then $\pi_{m}^{d *}<\pi_{m}^{r *}$ and $\pi_{r}^{d *}<\pi_{r}^{r s *}$.

Proof. See Appendix.

Theorem 2 shows that RS improves the profit of the manufacturer and the retailer in the decentralized supply chain when the RS coefficient $\varphi$ is in a certain threshold, that is, the manufacturer and the retailer are willing to implement the RS contract if and only if the RS coefficient satisfies $\left(\left[2 \eta-(t+\beta)^{4}\right]^{2} / 4 \eta^{2}\right)<\varphi<1$.

\section{Numerical Analysis}

From the aforementioned explanations, we are unable to obtain closed-form conditions for profit comparison considering various factors. So, we conduct a set of numerical experiments to study the factors how various factors, such as the CER cost factor $\eta$, the government's carbon tax $t$, and the consumers' low-carbon preference $\beta$ influence the manufacturer's CER decision, the retailer's profit, and the manufacturer's profit under RS. The parameters in this section were assumed from a previously published paper [5]; the parameter values are $a=20, c=4, \beta=1.2, e_{0}=10, t=0.2$, $\eta=6$, and $\varphi=0.6$. And the proposed model was solved by using MATLAB software. See Figures 1-6 for the decision variables with optimum values according to the parameter values.

5.1. Effect of $\eta$ on the Equilibrium Outcomes. Now, we explore how the CER cost factor $\eta$ influences the CER degree. Figure 1 shows that the CER degree is the greatest in the centralized system, medium under RS, and the lowest in the decentralized system. Moreover, when the CER cost factor decreases, the CRE degree increases at different rates, and the gap of the CER degree among these three scenarios would widen further. This suggests that the benefits of vertical integration increase rapidly with lower CER cost factor. And it means that RS produces a better effect to motivate the manufacturer's CER efforts with less CER cost factor.

Figure 2 illustrates that the manufacturer's and the retailer's profit increase with the decrease in the CER cost factor because a higher CER cost would increase the manufacturer's marginal cost. The manufacturer sets a higher wholesale price, and the retailer sets a higher retail price gradually. Consequently, the demand decreases, and the marginal profit becomes lower. Thus, the total optimal profit decreases due to a higher CER cost factor.

5.2. Effect of t on the Equilibrium Outcomes. Now, we explore how the carbon tax taffects the CER degree and supply chain members' profit. It can be seen from Figure 3 that the CER degree initially increases and then decreases with the carbon $\operatorname{tax} t$. It also says that there exists an optimal carbon tax for the government to spur the manufacturer to exert his best effort to curb carbon emission. The government should impose the highest carbon tax in the centralized system, medium under RS, and the lowest in the decentralized system.

5.3. Effect of $\beta$ on the Equilibrium Outcomes. Now, we explore how the customers' low-carbon preference $\beta$ affects the retail price. Figure 4 shows that the retailer should set the highest retail price in the decentralized system, medium under RS, and the lowest in the centralized system when $\beta$ is lower than the threshold. An intuitive explanation for this finding is that when $\beta$ is small, customers are far less likely to pay for lowcarbon products; thus, it is similar to the traditional supply chain situation. The manufacturer would set a lowest wholesale price in the centralized system, medium under RS, and the highest in the decentralized system, which yields his maximum profit. So, the retailer should set higher selling price to sustain his maximum profit compared to the centralized system. When $\beta$ goes beyond the certain threshold value, customers prefer to pay for low-carbon products. The retailer should set a highest retail price in the centralized system, medium under RS, and the lowest in the decentralized system. The reason is that when customers' lowcarbon preference is high, the positive influence on consumers' purchase decision-making would be obvious. More customers are willing to pay premiums for low-carbon products. Thus, the product's marginal profit becomes higher. The retailer should raise the selling price to improve the profit.

Figure 5 illustrates that the manufacturer's and the retailer's profit increase with the increase in the customers' low-carbon preference. A higher customers' low-carbon preference increases the customers' willingness to pay for low-carbon products, which makes the demand to increase and improves the manufacturer's and the retailer's profit accordingly.

5.4. Effect of $R S$ on the Equilibrium Outcomes. Now, we explore how RS affects the supply chain efficiency $E$. The optimal supply chain efficiency in the decentralized case $\left(E^{d *}=\left(\pi_{s c}^{d *} / \pi_{s c}^{c *}\right)\right)$ and under $\mathrm{RS}\left(E^{r s *}=\left(\pi_{s c}^{r s *} / \pi_{s c}^{c *}\right)\right)$ is described in Figure 6.

Figure 6 shows that the supply chain efficiency under RS is higher than that in the decentralized supply chain. The supply chain efficiency in the RS and decentralized scenario decreases with $\beta$ and $t$ but increases with $\eta$. This can be explained as follows. On the one hand, for given $\eta$, the rise of 


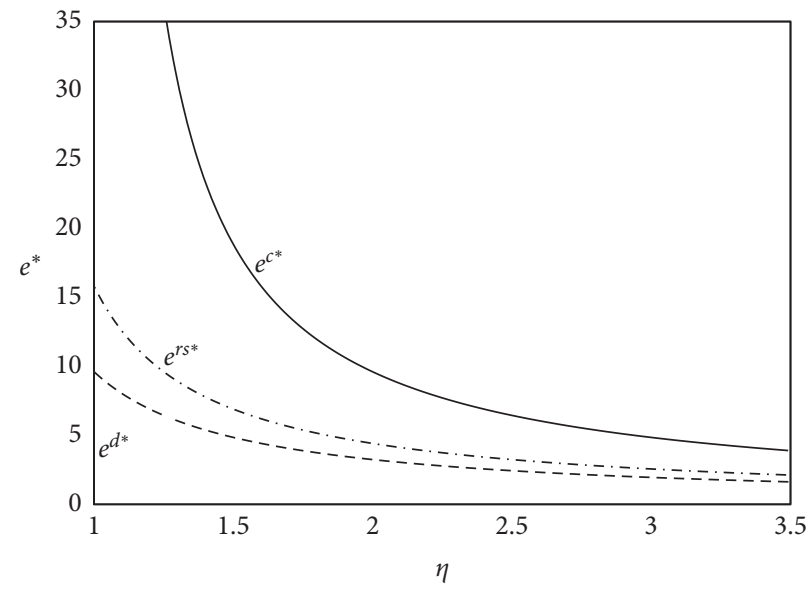

FIGURE 1: Effect of $\eta$ on the CER degree.

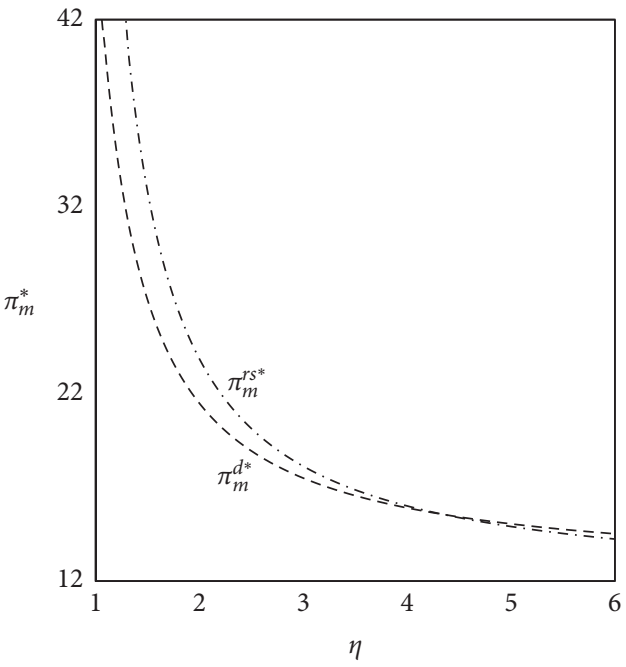

(a)

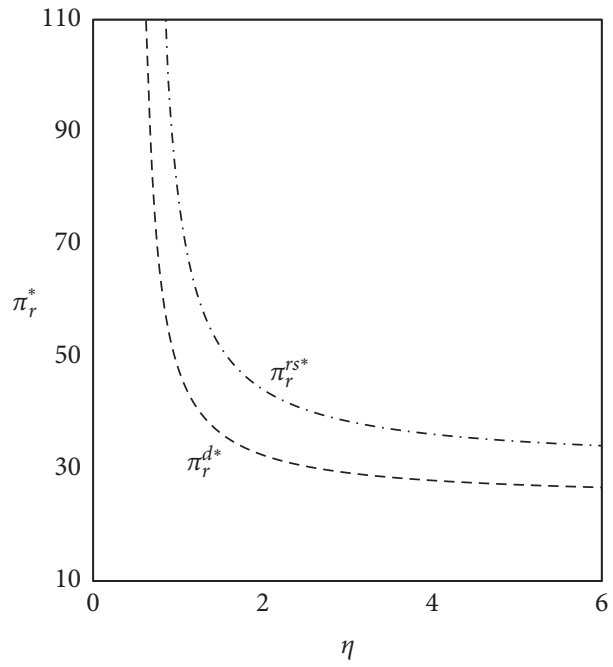

(b)

FIGURE 2: Effect of $\eta$ on the manufacturer and retailer's profit.

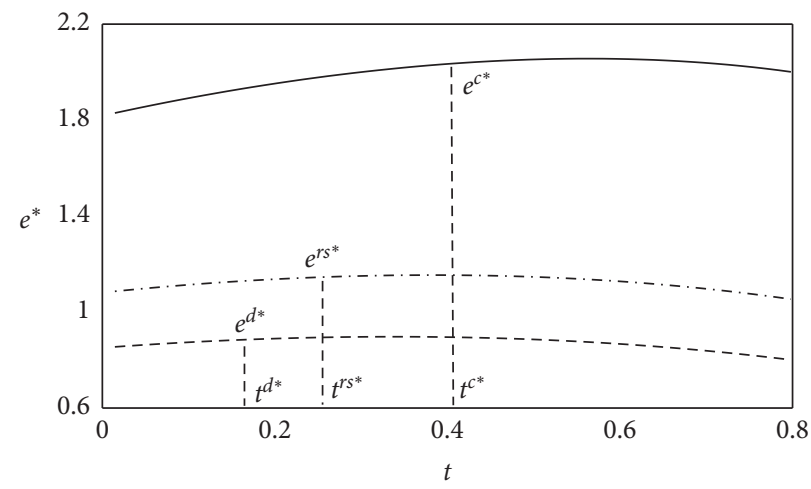

Figure 3: Effect of $t$ on the manufacturer and retailer's profit.

the product's marginal profit enlarges as $\beta$ and $t$ increase; thus, the manufacturer exerts more effort in CER, which intensifies double marginalization and thus worsens the channel efficiency. On the other hand, for given $\beta$ and $t$, the expansion of the product's marginal profit diminishes when $\eta$ escalates; then, the manufacturer makes less effort in CER, which counteracts double marginalization and eventually improves the supply chain efficiency. 


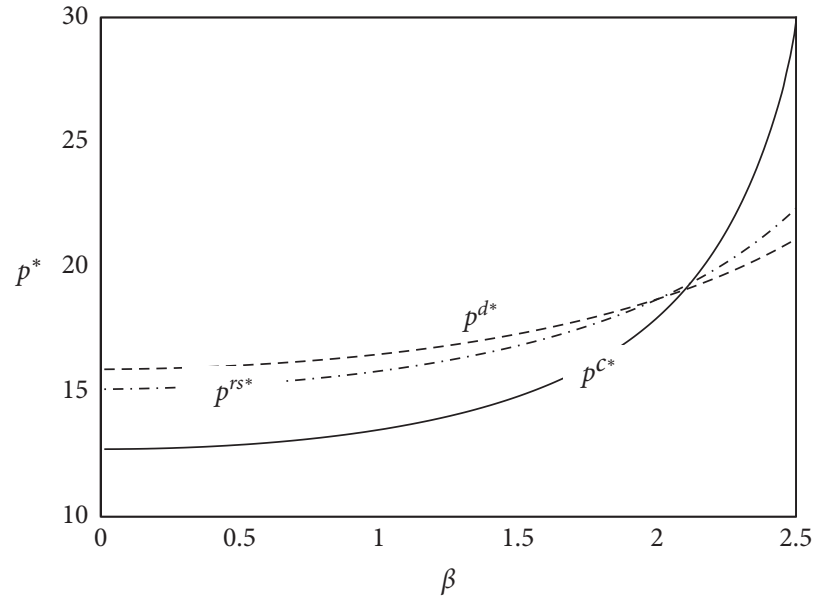

FIgURE 4: Effect of $\beta$ on the retail price.

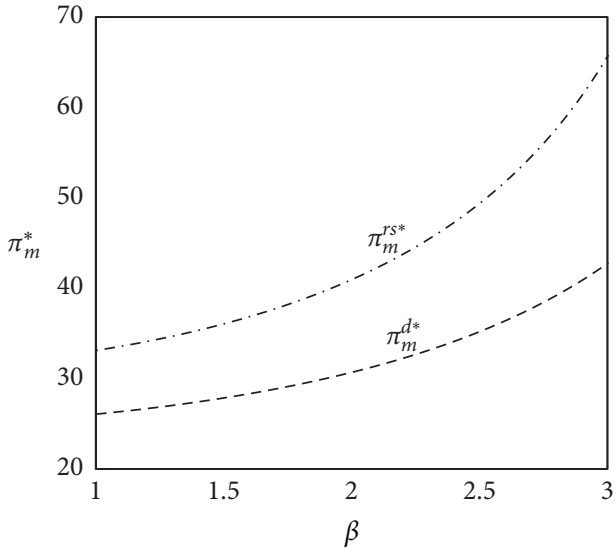

(a)

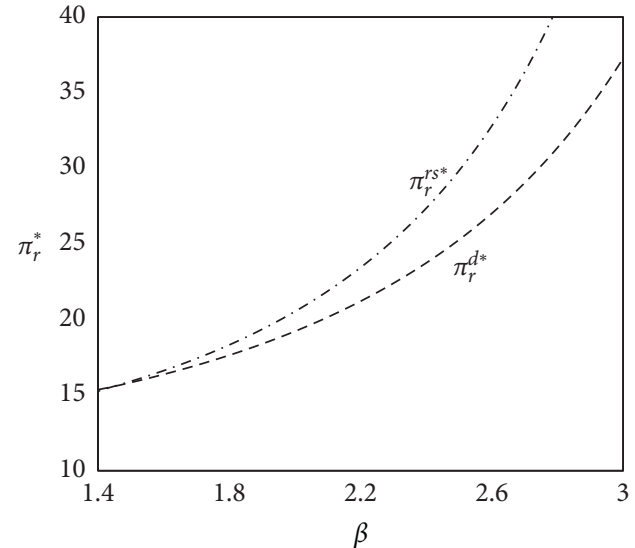

(b)

Figure 5: Effect of $\beta$ on the manufacturer and retailer's profit.

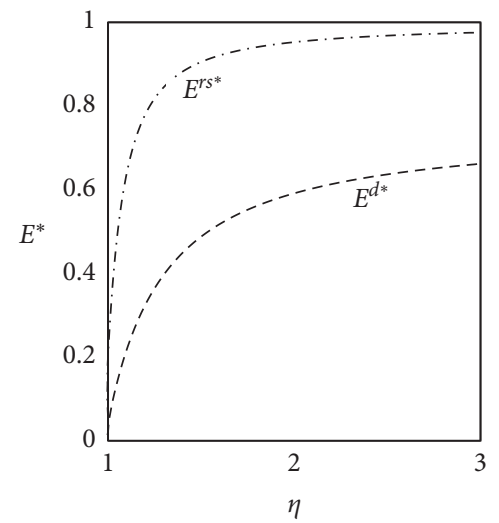

(a)

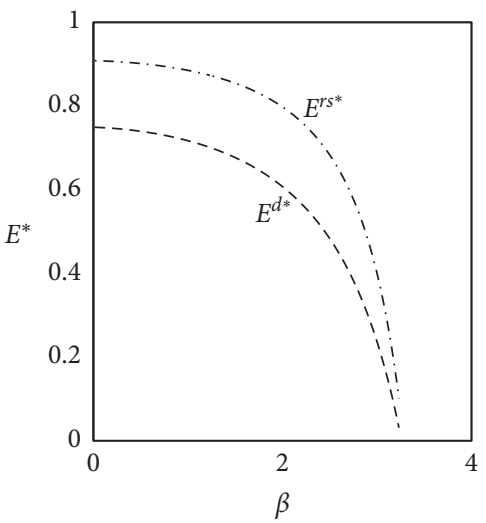

(b)

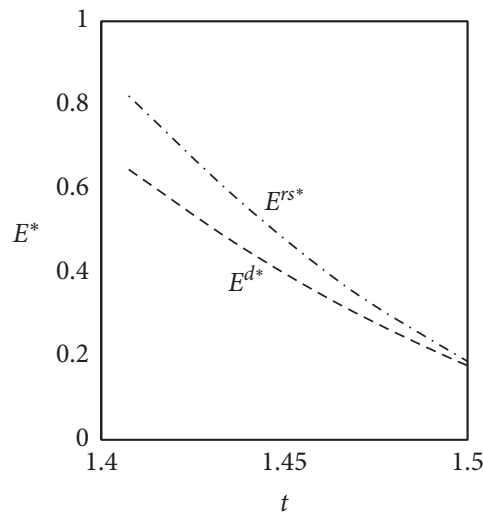

(c)

FIGURE 6: Impact of RS on the supply chain efficiency. 


\section{Managerial Implication}

This paper demonstrates some key and deliberate insights for managers and policy makers who need to consider the manufacturer's CER cost factor, the government's carbon tax, and consumers' low-carbon preference. The managerial implication for managers and policy makers can be obtained as follows:

(1) With the decrease in the CER cost factor, the CER degree and the supply chain members' profits will increase. Therefore, the manufacturer should improve the CER degree with the decrease in the CER cost factor. Moreover, the manufacturer should set the highest CER degree in the centralized, medium under $\mathrm{RS}$, and the lowest in the decentralized supply chain.

(2) As consumers' low-carbon preference increases, the retail price and the supply chain members' profits increase significantly. Therefore, the retailer should improve the retail price with the increase in the consumers' low-carbon preference. Moreover, the retailer should set the highest selling price in the decentralized, medium under RS, and the lowest in the centralized supply chain when $\beta$ is below a certain threshold, and the retailer should set the highest selling price in the centralized, medium under RS, and the lowest in the decentralized supply chain when $\beta$ is below a certain threshold.

(3) Carbon tax has a certain incentive effect on the manufacturer's CER degree. The government should impose different carbon taxes in different scenarios, i.e., the highest carbon tax in the centralized supply chain, medium under RS, and the lowest in the decentralized supply chain. Based on the optimal carbon tax imposed by the government, the incentive mechanism can stimulate the manufacturer to increase their efforts towards CER investment for environmental and economic benefits.

(4) RS cannot perfectly coordinate the supply chain but can improve the supply chain efficiency and encourage the manufacturer to increase CER investment.

\section{Conclusion}

In this study, some factors are taken into consideration such as the manufacturer's CER cost factor, the government's carbon tax, and consumers' low-carbon preference to establish profit models of the low-carbon supply chain. Stackelberg game models are developed to address the centralized, decentralized, and RS scenarios between the manufacturer and the retailer. The models determine the optimal CER degree and price decisions, as well as the carbon tax imposed by the government. This paper finds that the supply chain would benefit from the increment of consumer's environmental awareness but be depressed by the increase of CER cost. Additionally, there exists a unique optimal carbon tax to improve CER degree in different scenarios. Furthermore, this paper finds that RS is effective to inspire the manufacture to exert the best efforts to improve CER degree. Moreover, RS is a feasible incentive tool to the manufacturer and retailer when the RS coefficient is in a certain threshold value.

There are some limitations on this paper. The assumption is that the manufacturer is in the dominant position in the supply chain. And this paper adopts simulated data to verify the proposed model. A case study utilizing real industrial data is not inserted in this model. So, retailer-dominant or/and powerbalanced scenarios should be discussed in the future. And a case study utilizing real industrial data should be extended. These extensions will help us come to a better understanding on the low-carbon supply chain operation in the future [27, 28].

\section{Appendix}

Proof of Proposition 1. The Hessian matrix of $\pi_{s c}^{c *}$ $H_{1}=\left[\begin{array}{cc}\left(\partial^{2} \pi_{m} / \partial e^{2}\right) & \left(\partial^{2} \pi_{m} / \partial e \partial p\right) \\ \left(\partial^{2} \pi_{m} / \partial p \partial e\right) & \left(\partial^{2} \pi_{m} / \partial p^{2}\right)\end{array}\right]=\left[\begin{array}{cc}-2 & \beta-t \\ \beta-t & 2 \beta t-\eta\end{array}\right]$, which is negatively definite if $\left|H_{1}\right|=2 \eta-(\beta+t)^{2}>0 . \pi_{s c}^{C}$ is a strictly joint convex function of eand $p$, and there is an optimal solution.

Solving the first-order conditions $\left(\partial \pi_{s c}^{c} / \partial e\right)=0$ and $\left(\partial \pi_{s c}^{c} / \partial p\right)=0$ for $(p, e)$, we obtain the optimal CER degree $e^{c *}$ and retail price $p^{c *}$, respectively, in Proposition 1 . To ensure $e^{c *}>0$, we assume that $a-c-t e_{0}>0$ throughout this paper.

Substituting $e^{c *}$ and $p^{c *}$ into (1), we obtain the maximum supply chain's profit $\pi_{s c}^{c *}$ in Proposition 1 . To ensure the profit is positive, we assume that $(\beta+t)^{2}<2 \eta$ throughout the paper.

Proof of Lemma 1.

(1)

(i) Taking the first derivative of $e^{c *}$ with respect to $\eta$, we obtain

$$
\begin{aligned}
\frac{\partial e^{c *}}{\partial \eta} & =\frac{-2(\beta+t)\left(a-c-t e_{0}\right)}{\left[2 \eta-(\beta+t)^{2}\right]^{2}} ; \text { if } a-c-t e_{0} \\
& >0, \text { then } \frac{\partial e^{c *}}{\partial \eta}<0 .
\end{aligned}
$$

(ii) Taking the first derivative of $p^{c *}$ with respect to $\eta$, we obtain

$$
\begin{aligned}
\frac{\partial p^{c *}}{\partial \eta} & =\frac{\left(a-c-t e_{0}\right)\left(t^{2}-\beta^{2}\right)}{\left[2 \eta-(\beta+t)^{2}\right]^{2}} ; \text { if } a-c-t e_{0} \\
& >0 \text { and } t<\beta, \text { then } \frac{\partial p^{c *}}{\partial \eta}<0 .
\end{aligned}
$$

(iii) Similarly, $\left(\partial \pi_{s c}^{c *} / \partial \eta\right)=\left(2 \eta(\beta+t)\left(c-a+e_{0} t\right)^{2}\right.$ $\left./ 2\left[(\beta+t)^{2}-2 \eta\right]^{2}\right)<0$.

(2)

(i) Taking the first derivative of $e^{c *}$ with respect to $\beta$, we obtain 


$$
\begin{aligned}
\frac{\partial e^{c *}}{\partial \beta} & =\frac{\left[2 \eta+(\beta+t)^{2}\right]\left(a-c-t e_{0}\right)}{\left[2 \eta-(\beta+t)^{2}\right]^{2}} ; \text { if } a-c-t e_{0} \\
& >0 \text {, then } \frac{\partial e^{c *}}{\partial \beta}>0 .
\end{aligned}
$$

(ii) Taking the first derivative of $p^{c *}$ with respect to $\beta$, we obtain $\left(\partial p^{c *} / \partial \beta\right)=\left(\left(a-c-t e_{0}\right)[2 \eta \beta-t\right.$ $\left.\left.(\beta+t)^{2}\right] /\left[2 \eta-(\beta+t)^{2}\right]^{2}\right)$. $a-c-t e_{0}>0, t<\beta$, and $(\beta+t)^{2}<2 \eta$, then $\left(\partial p^{c *} / \partial \beta\right)>0$.

(iii) Similarly, $\left(\partial \pi_{s c}^{c *} / \partial \beta\right)=\left(2 \eta(\beta+t)\left(c-a+e_{0} t\right)^{2}\right.$ $\left./ 2\left[(\beta+t)^{2}-2 \eta\right]^{2}\right)>0$.

(3) Taking the second derivative of $e^{c *}$ with respect to $t$, we obtain $\left(\partial^{2} e^{c *} / \partial t^{2}\right)=-e_{0} \eta-2<0$, that is, $e^{c *}$ is a convex function of $t$. Solving the first-order condition $\left(\partial e^{c *} / \partial t\right)=\left(\left(c-a+\beta e_{0}+2 e_{0} t\right) /(\beta+t)^{2}-2 \eta\right)$ $-\left(\begin{array}{llll}2 & (\beta+t) & \left(\beta c-a \beta-a t+c t+e_{0} t^{2}+\beta e_{0} t\right) /[(\beta+\end{array}\right.$ $\left.t)^{2}-2 \eta\right]^{2}$ ) $=0$ for $t$, we can obtain the optimal carbon tax $t^{c *}$.

Proof of Proposition 2. Taking the second derivative of $\pi_{r}^{d}$ with respect to $p$, we obtain that $\left(\partial^{2} \pi_{r}^{d} / \partial p^{2}\right)=-e_{0} \eta-2<0$, i.e., $\pi_{r}^{d}$ is a convex function of $p$. Solving the first-order condition for $p$, we can derive the retail price reaction function:

$$
p(w, e)=\frac{a+\beta e+w}{2} .
$$

Substituting (A 4) into (4), we obtain that

$$
\pi_{m}^{d}=\frac{1}{2}\left[w-c-t\left(e_{0}-e\right)\right](a+\beta e-w)-\frac{1}{2} \eta e^{2} .
$$

According to equation (A 5), the Hessian matrix of $\pi_{m}^{d}$ $H_{2}=\left[\begin{array}{cc}\left(\partial^{2} \pi_{m}^{d} / \partial e^{2}\right) & \left(\partial^{2} \pi_{m}^{d} / \partial e \partial w\right) \\ \left(\partial^{2} \pi_{m}^{d} / \partial w \partial e\right) & \left(\partial^{2} \pi_{m}^{d} / \partial w^{2}\right)\end{array}\right]$

$\left[\begin{array}{cc}t \beta-\eta & (1 / 2) \beta-(1 / 2) t \\ (1 / 2) \beta-(1 / 2) t & -1\end{array}\right]$, which is negatively definite if $2 \eta-(t+\beta)^{2}>0, t \beta-\eta>0$. So, $\pi_{m}^{d}$ is a strictly joint convex function of $e$ and $w$. Solving the first-order conditions, we obtain the optimal CER degree $\left(e^{d *}\right)$ and wholesale price $\left(w^{d *}\right)$ in Proposition 2. Substituting $e^{d *}, w^{d *}$ into (4)-(A 4), we obtain the optimal retail price $\left(p^{d *}\right)$, the maximum supply chain members' profits $\left(\pi_{m}^{d *}, \pi_{r}^{d *}\right)$, and the maximum channel's profit $\left(\pi_{s c}^{d *}\right)$ in Proposition 2.

\section{Proof of Lemma 2.}

(i) Taking the first derivative of $e^{d *}$ with respect to $\eta$, we obtain $\left(\partial e^{d *} / \partial \eta\right)=(-4(\beta+t)(a-c-$ $\left.\left.t e_{0}\right) /\left[4 \eta-(\beta+t)^{2}\right]^{2}\right)$. If $a-c-t e_{0}>0$, then $\left(\partial e^{d *} / \partial \eta\right)<0$. (ii) Taking the first derivative of $p^{d *}$ with respect to $\eta$, we obtain $\left(\partial p^{d *} / \partial \eta\right)=((c-$ $\left.\left.a+t e_{0}\right)(3 \beta-t)(\beta+t) / \quad\left[4 \eta-(\beta+t)^{2}\right]^{2}\right)$. If $c-a+t e_{0}<0, \beta>t$, then $\left(\partial p^{d *} / \partial \eta\right)<0$.

(iii) Taking the first derivative of $\pi_{s c}^{d *}$ with respect to $\eta$, we obtain $\left(\partial \pi_{s c}^{d *} / \partial \eta\right)=$ $-\left((\beta+t)^{2} \quad\left(c-a+e_{0} t\right)^{2}\left[(\beta+) t^{2}-8 \eta\right] / 2[(\beta+\right.$ $\left.\left.t)^{2}-4 \eta\right]^{3}\right)$. If $(\beta+t)^{2}<2 \eta$, then $\left(\partial \pi_{s c}^{d *} / \partial \eta\right)<0$.

(2)

(i) Taking the first derivative of $e^{d *}$ with respect to $\beta$, we obtain $\left(\partial e^{d *} / \partial \beta\right)=\left(-\left[4 \eta+(\beta+t)^{2}\right]\right.$ $\left.\left(c-a+t e_{0}\right) /\left[4 \eta-(\beta+t)^{2}\right]^{2}\right)$. And if $c-a+$ $t e_{0}<0$, then $\left(\partial e^{d *} / \partial \beta\right)>0$.

(ii) Taking the first derivative of $p^{d *}$ with respect to $\beta$, we obtain $\left(\partial p^{d *} / \partial \beta\right)=\left(\left(c-a+e_{0} t\right)[t(\beta+\right.$ $\left.\left.t)^{2}-2 \eta(3 \beta+t)\right] /\left[(\beta+t)^{2}-4 \eta\right]^{2}\right)$. And if $c-a+t e_{0}<0, t<\beta$, and $(\beta+t)^{2}<2 \eta$, then $\left(\partial p^{d *} / \partial \beta\right)>0$.

(iii) Taking the first derivative of $\pi_{s c}^{d *}$ with respect to $\beta$, we obtain $\left(\partial \pi_{s c}^{d *} / \partial \beta\right)=\left(\eta(\beta+t)\left(c-a+t e_{0}\right)^{2}\right.$ $\left.\left[(\beta+t)^{2}-8 \eta\right] /\left[(\beta+t)^{2}-4 \eta\right]^{3}\right)$. And if $c-a$ $+t e_{0}<0$ and $(\beta+t)^{2}<2 \eta$, then $\left(\partial \pi_{s c}^{d *} / \partial \beta\right)>0$.

(3) Taking the second derivative of $e^{d *}$ with respect to $t$, we obtain $\left(\partial^{2} e^{d *} / \partial t^{2}\right)<0$, i.e., $e^{d *}$ is a convex function of $t$. Taking the first derivative of $e^{d *}$ with respect to $t$, we obtain $\left(\partial e^{d *} / \partial t\right)=\left(\left(c-a+\beta e_{0}+\right.\right.$ $\left.\left.2 e_{0} t\right) /(\beta+t){ }^{2}-4 \eta\right)-\left(2(\beta+t)^{2}\left(c-a+e_{0} t\right) /[(\beta+\right.$ $\left.\left.t)^{2}-4 \eta\right]^{2}\right)$. Solving the first-order conditions, we can obtain the optimal carbon $\operatorname{tax} t^{d *}$.

\section{Proof of Theorem 1.}

(1)

(i) If $0<\varphi<1$, it is obvious that $e^{c *}-e^{d *}=((a-$ $\left.\left.c-t e_{0}\right)(\beta+t) / 2 \eta-(\beta+t)^{2}\right)-\left(\left(a-c-t e_{0}\right)\right.$ $\left.(\beta+t) / 4 \eta-(\beta+t)^{2}\right)>0$

(ii) If $(\beta+t)^{2}<2 \eta, \quad$ it is obvious that $\pi_{s c}^{c *}-\pi_{s c}^{d *}=\left(-\eta\left(c-a+e_{0} t\right)^{2} / 2\left[(\beta+t)^{2}-2 \eta\right]\right)+$ $\left(\eta\left(c-a+e_{0} t\right)^{2}\left[(\beta+t)^{2}-6 \eta\right] / 2\left[(\beta+t)^{2}-4 \eta\right]^{2}\right)$ $>0$

(iii) If $\eta<t \beta+\beta^{2}$, it is obvious that $p^{c *}-p^{d *}=$ $\left(\left(a+c+t e_{0}\right)(\eta-\beta t)-\beta^{2} c-\beta^{2} t e_{0}-t^{2} a / 2 \eta-\right.$ $\left.(\beta+t)^{2}\right)-\left(\left(a+c+t e_{0}\right)(\eta-\beta t)-\beta^{2} c-\beta^{2} t\right.$ $\left.e_{0}-t^{2} a+2 a \eta / 4 \eta-(\beta+t)^{2}\right)>0$; if $\eta>t \beta+\beta^{2}$, $p^{c *}-p^{d *}<0$; if $\eta=t \beta+\beta^{2}, p^{c *}=p^{d *}$

Proof of Theorem 2.

(i) If $0<\varphi<1$, it is easy to prove that $e_{m}^{d *}<e_{m}^{r * *}$.

(ii) If $\pi_{m}^{d *}<\pi_{m}^{r s *}$, then $0<\varphi<1$; if $\pi_{r}^{d *}<\pi_{r}^{r s *}$, then $\left(\left[2 \eta-(t+\beta)^{4}\right]^{2} / 4 \eta^{2}\right)<\varphi<1$. So, if $\quad([2 \eta-(t+$ $\left.\left.\beta)^{4}\right]^{2} / 4 \eta^{2}\right)<\varphi<1$, we obtain $\pi_{m}^{d}<\pi_{m}^{r s}$ and $\pi_{r}^{d}<\pi_{r}^{r s}$.

\section{Data Availability}

The underlying data supporting the results of this study can be found in the manuscript. 


\section{Conflicts of Interest}

The authors declare that there are no conflicts of interest regarding the publication of this paper.

\section{Acknowledgments}

This work was supported by Academic Discipline Project of Shanghai Dianji University (Project no.16Ysxk03), Special Program for Humanities and Social Science of Shanghai Dianji University, and the National Social Science Fund of China, "Research on the Practical Orientation of Green Consumption in the New Era" (Project no. 20BKS079).

\section{References}

[1] S. Du, J. Zhu, H. Jiao, and W. Ye, "Game-theoretical analysis for supply chain with consumer preference to low carbon," International Journal of Production Research, vol. 53, no. 12, pp. 3753-3768, 2015.

[2] D. W. Thompson, R. C. Anderson, E. N. Hansen, and L. R. Kahle, "Green segmentation and environmental certification: insights from forest products," Business Strategy and the Environment, vol. 19, no. 5, pp. 319-334, 2010.

[3] H. Zhang, L. Li, P. Zhou, J. Hou, and Y. Qiu, "Subsidy modes, waste cooking oil and biofuel: policy effectiveness and sustainable supply chains in China," Energy Policy, vol. 65, pp. 270-274, 2014.

[4] H. Peng, T. Pang, and J. Cong, "Coordination contracts for a supply chain with yield uncertainty and low-carbon preference," Journal of Cleaner Production, vol. 205, pp. 291-302, 2018.

[5] H. Yang and W. Chen, "Retailer-driven carbon emission abatement with consumer environmental awareness and carbon tax: revenue-sharing versus Cost-sharing," Omega, vol. 78, pp. 179-191, 2018.

[6] S. Chen, X. Wang, Y. Wu, and F. Zhou, "Pricing policies of a dual-channel supply chain considering channel environmental sustainability," Sustainability, vol. 9, no. 3, p. 382, 2017.

[7] T. Li, R. Zhang, S. Zhao, and B. Liu, "Low carbon strategy analysis under revenue-sharing and cost-sharing contracts," Journal of Cleaner Production, vol. 212, pp. 1462-1477, 2019.

[8] Y. Zhou, F. Hu, and Z. Zhou, "Pricing decisions and social welfare in a supply chain with multiple competing retailers and carbon tax policy," Journal of Cleaner Production, vol. 190, pp. 752-777, 2018.

[9] Y. Cheng, Z. Xiong, and Q. Luo, "Joint pricing and product carbon footprint decisions and coordination of supply chain with cap-and-trade regulation," Sustainability, vol. 10, no. 2, p. $481,2018$.

[10] H. Zhang, Z. Zhang, X. Pu, and Y. Li, "Green Manufacturing Strategy Considering Retailers' Fairness Concerns," Sustainability, vol. 11, no. 17, p. 4646, 2019.

[11] Y. Wang, W. Chen, and B. Liu, "Manufacturing/remanufacturing decisions for a capital-constrained manufacturer considering carbon emission cap and trade," Journal of Cleaner Production, vol. 140, pp. 1118-1128, 2017.

[12] Q. Li, T. Xiao, and Y. Qiu, "Price and carbon emission reduction decisions and revenue-sharing contract considering fairness concerns," Journal of Cleaner Production, vol. 190, pp. 303-314, 2018.
[13] H. Yang, J. Luo, and H. Wang, “The role of revenue sharing and first-mover advantage in emission abatement with carbon tax and consumer environmental awareness," International Journal of Production Economics, vol. 193, pp. 691-702, 2017.

[14] A. A. Taleizadeh, N. Alizadeh-Basban, and B. R. Sarker, "Coordinated contracts in a two-echelon green supply chain considering pricing strategy," Computers \& Industrial Engineering, vol. 124, pp. 249-275, 2018.

[15] D. Ghosh and J. Shah, "Supply chain analysis under green sensitive consumer demand and cost sharing contract," International Journal of Production Economics, vol. 164, pp. 319-329, 2015.

[16] P. He, Y. He, C. Shi, H. Xu, and L. Zhou, "Cost-sharing contract design in a low-carbon service supply chain," Computers \& Industrial Engineering, vol. 139, Article ID 106160, 2020.

[17] U. Mishra, J.-Z. Wu, and B. Sarkar, "Optimum sustainable inventory management with backorder and deterioration under controllable carbon emissions," Journal of Cleaner Production, vol. 279, Article ID 123699, 2021.

[18] W. Chen and Z.-H. Hu, "Using evolutionary game theory to study governments and manufacturers' behavioral strategies under various carbon taxes and subsidies," Journal of Cleaner Production, vol. 201, pp. 123-141, 2018.

[19] Q. Wang, D. Zhao, and L. He, "Contracting emission reduction for supply chains considering market low-carbon preference," Journal of Cleaner Production, vol. 120, pp. 7284, 2016.

[20] Y. Wu, R. Lu, J. Yang, F. Xu, and C. Services, "Low-carbon decision-making model of online shopping supply chain considering the $\mathrm{O} 2 \mathrm{O}$ model," Journal of Retailing and Consumer Services, vol. 59, Article ID 102388, 2021.

[21] L. Liang and L. Futou, "Differential game modelling of joint carbon reduction strategy and contract coordination based on low-carbon reference of consumers," Journal of Cleaner Production, vol. 277, Article ID 123798, 2020.

[22] X. Wang, M. Xue, and L. Xing, "Analysis of carbon emission reduction in a dual-channel supply chain with cap-and-trade regulation and low-carbon preference," Sustainability, vol. 10, no. 3, p. 580, 2018.

[23] B. Yu, J. Wang, X. Lu, and H. Yang, "Collaboration in a lowcarbon supply chain with reference emission and cost learning effects: cost sharing versus revenue sharing strategies," Journal of Cleaner Production, vol. 250, 2020.

[24] G. Liu, H. Yang, and R. Dai, "Which contract is more effective in improving product greenness under different power structures: revenue sharing or cost sharing?" Computers \& Industrial Engineering, vol. 148, Article ID 106701, 2020.

[25] Z. Liu, T. D. Anderson, and J. M. Cruz, "Consumer environmental awareness and competition in two-stage supply chains," European Journal of Operational Research, vol. 218, no. 3, pp. 602-613, 2012.

[26] D. Yang and T. Xiao, "Pricing and green level decisions of a green supply chain with governmental interventions under fuzzy uncertainties," Journal of Cleaner Production, vol. 149, pp. 1174-1187, 2017.

[27] Q. Li, M. Shi, and Y. Huang, "A dynamic price game model in a low-carbon, closed-loop supply chain considering return rates and fairness concern behaviors," International Journal of Environmental Research and Public Health, vol. 16, no. 11, p. 1978, 2019.

[28] T. Li, R. Zhang, S. Zhao, and B. Liu, "Low carbon strategy analysis under revenue-sharing and cost-sharing contracts," Journal of Cleaner Production, vol. 212, pp. 1462-1477, 2019. 